\title{
Novel Methodology for Crop Disease Detection
}

\author{
Asit Dhaware ${ }^{1}$, Akash Fulmali ${ }^{2}$, Sahil Hatankar ${ }^{3}$, Dr. Sashikala Mishra ${ }^{4}$, Prof. Prashant Gadakh ${ }^{5}$ \\ International Institute of Information Technology, Pune ${ }^{1,2,3,4,5}$ \\ Department of Computer Engineering ${ }^{1,2,3,4,5}$
}

\begin{abstract}
Our country's economy is something, which is highly depends on the agricultural productivity. Due to this reason the disease detection in agricultural field has become one of the most important factors as it plays an important role for successful cultivation .Even though the technology has emerged periodically, due to some reasons traditional methodologies are being used. Due to usage of traditional methodologies , the plant diseases are wrongly identified which leads to huge loss of time, money, productivity, quality, and quantity of the cultivated crops .Now a days, expertise use manual prediction methodology ,but for this large number of experts are required and it also requires continuous monitoring of plants, due to which its cost is very high for a middle class farmer .Generally, by observing the symptoms on the leaves of a plant, we can predict the diseases of a plant.
\end{abstract}

Keywords: Neural networks, CNN, Machine learning, Crop diseases, Disease detection, Diagnosis.

\section{INTRODUCTION}

Worldwide, crop diseases cause major production loss and economical loss in agricultural industry. For agriculture to sustain, it is impossible for a farmer to monitor the health of plants or trees and to detect their diseases. In the current time, there are no sensors available for real-time assessment of health situation in trees on commercial basis. Diagnosis of the diseases based on the detection of symptoms occurred earlier, has been a usual threshold taken into account for integrated pest management strategies. Earlier, sanitary treatment with regard to pests and pathogens has been a great procedure to minimize crops yield losses and to increase the efficiency and efficacy of the treatments. However, there are complications in early identification, which delays the application of the appropriate corrective actions due to the possibility of new diseases associated with new resistant crop variants. Earlier, detection of diseases among farmers and technicians can be leveraged by the use of image-based automated identification systems, but under real field conditions they perform poorly using smart phone devices [7].

By observable patterns of specific plant, the studies of plant or fruit can be determined. But, it is very difficult to detect disease and monitor health of the plant. Pesticides, fungicides and chemical applications, using such proper management strategies, one can facilitates the control of diseases which interns improved quality [8].In recent years, with the development of computational systems, and in particular Machine Learning-related Artificial Intelligence applications, Graphical Processing Units (GPUs) embedded processors, IOTs, Cloud Computing have achieved exponential growth in technology, leading to the development of application models and novel methodologies [9].

\section{LITERATURE SURVEY}

In March 2011, Authors Heba Al-Hiary, Mohamad Hashem experimentally proposed and provided a software solution for fast and more accurate detection and classification of plant diseases. After the segmentation, successively two steps were added. The identification of mostly green pixels are done in the first step. Based on the specific threshold values, these pixels are masked in second step. By using Otsu's method these threshold values are computed, then those mostly green pixels are masked. Complete removal of pixels with zeros red, green and blue values and the pixels on the boundaries of infected object was the another addition step. The proposed technique is a one of the robust technique for the detection of plant leaves diseases, was demonstrated by the results of this experiment. The developed algorithm's efficiency would successfully detect and classify the examined diseases with a precision between $83 \%$ and $94 \%$ [1].

In 30 may 2014, Author Emanuel Cortes proposed a paper "Plant Disease Classification Using Convolution Networks and Generative Adversarial Networks". As per author, due to the growth of Smartphone technology throughout the globe, it has now become technically feasible to leverage image processing techniques to identity type of plant disease by capturing a simple photo. To reduce the effects of crop diseases on food supply, quicker interventions are implemented that can be achieve by identifying disease. A public dataset of 86,147 images of diseased and healthy plants are used, a deep convolution network and semi supervised methods 


\section{International Journal of Research in Advent Technology, Vol.7, No.2, February 2019 E-ISSN: 2321-9637 \\ Available online at www.ijrat.org}

are trained for classification of crop species and disease status of 57 different classes[2].

In 29 January 2016, Authors Jayme Garcia, Arnal Barbedo proposed a review on main challenges in automatic plant diseases identification based on visible range images. When system tried to identify disease using simple image, many problems had occurred during this. In this paper, the problems or challenges that faced by system are given. Extrinsic factors and Intrinsic factors are specified. Image background and image captured conditions were the extrinsic factors addressed. Intrinsic factor contains system segmentation, multiple simultaneous disorders and different disorders with similar symptoms. Real time operation, computational power etc. are the other challenges that were identified. The problems and their possible solutions were also given[3].

In 29 May 2016, Authors Srdjan Sladojevic, Marko Arsenovic, Andras Anderla, Dubravko Culibrk, and Darko Stefanovic proposed a software solution for leaf image classification using deep neural networks based recognition of plant diseases. Impressive results were achieved in the field of image classification by the convolution neural networks (CNNs). Based on leaf image classification, there has been development of plant disease recognition model, by the use of deep convolution networks which is concerned in this paper which has been a new approach. This developed model proposed by author can recognize different types of plant diseases out of healthy leaves, and system can easily remove noise. This is first time that CNN algorithm is used for plant disease identification. All necessary step which are required for developing model such as gathering images, creating database, training and testing models, etc. are described in this paper. "Caffe" (a deep learning framework) used for the deep CNN training. The developed model achieved accuracy between $91 \%$ and $98 \%$, when separate class tests carried then model gave average $96.3 \%$ accuracy [5].

A model using deep learning for image-based plant disease detection was proposed by the Authors Sharada P. Mohanty, David P. Hughes and Marcel Salathe in 22 September 2016. Plant disease detection using deep learning was the primary motive. Plant village dataset is used for training purpose. A dataset contained 54,306 images 14 crop species and 26 diseases were specified. There are two ways for building a model, transfer learning and training from scratch. For training purpose, AlexNet and GoogleNet deep learning architecture choosen. 5 different training and testing set distribution used; wherever Train: $80 \%$ and Test:20\% give high accuracy. According to result, Transfer learning way and GoogleNet architecture is highly efficient. An accuracy of $99.359 \%$ on a test set was achieved by the trained model. On field testing model accuracy is reduced to just above 31\%[4].

In March 2017, Author Suja radha proposed a solution for detection of leaf disease using image processing. In agricultural field, disease identification of plants has always been difficult. In this paper, they used image processing in MATLAB for identification of the disease. It included the following steps: 1) Loading the images, 2) Contrast enhancement, 3) Converting RGB to HSV, 4) Extracting of features and SVM. In this project, for feature extraction they used RGB, HSV, YIQ and Dithered Images. They used cotton leaf for experiment. For segmentation K-means clustering algorithm is used and SVM is used for classification[6]. In 21 april 2017, Authors Alexander Johannes, Artzai Picon, Aitor Alvarez-Gila, Jone Echazarra, Sergio Rodriguez-Vaamonde, Ana Díez Navajas proposed a paper "Automatic plant disease diagnosis using mobile capture devices applied on a wheat use case". Unlike other papers, this paper tries to focus on mainly early disease detection and multiple disease detection. Dataset were created using 7 mobile devices in field condition crop. The wheat is considered and mainly 3 disease septoria, rust and tan_spot, where more than 3500 images captured. In algorithm system extracted hotspot candidate, then analysed each hotspot in detail by local descriptors that extract and categorised each region to check against different disease using classifier. Obtained results in field condition higher than 0.30 for all the analysed diseases[7].

In Jan-2018, Authors Dixit Ekta Gajanan, Gavit Gayatri Shankar, Gode Vidya Keshav proposed a technique for plant disease identification in android based system using "Feature Extraction". For the recognition of plant diseases in the early stage, we can use intelligent systems instead of assigning professional agriculture engineers. The purpose of system is Supervising the diseases on crop and suggesting solution forgood productivity. The recognition of a disease can often be based on symptoms like lesions or spots in various parts of a plant. The color, area and the number of these spots on different parts of plant can determine to a great extent, the disease by which plant has been affected. If necessary higher cost molecular analyses and tests can be followed. This application can easily be extended for different plant diseases and different smart phone platforms [8].

In 12 Feb 2018, Authors Konstantinos P. ferentinos proposed a solution for plant disease detection and diagnosis using deep learning models. For this purpose, they used healthy and disease leaf images where total 


\section{Available online at www.ijrat.org}

87,848 images are used for 25 different plants disease identification. Best result is $99.53 \%$ with CNN (VGG Model). Based on the edges and lines in image, CNN algorithm can easily found disease. Plant diseases were identified using plantvillage database of leaves images of 14 different plants. As per paper, results were quite accurate using CNN. But there has been one drawback, that the images were solely from datasets, and no real photos were taken from cultivation field. They used $80 \%$ of images for training and $20 \%$ images for testing. Among all CNN models, VGG Model and AlexnetQWTBn architecture gave best success rate. Training time: 5.5 days [9].

In 22 April 2018, Authors, Andreas Kamilaris, Francesc $\mathrm{X}$. Prenafeta-Boldú surveyed on different technique and proposed a paper on it.With promising results and large potential, deep learning constitutes a recent modern technique for image processing and data analysis. After successfully applying deep learning in various domains, now it has entered into the domain of agriculture. A survey of 40 researches has given. The different data pre-processing techniques were examined. Comparison has been made between "Deep learning" and other existing popular techniques (with respect to differences in classification or regression). Results of the comparison clearly indicated that, deep learning provides high accuracy, outperforming existing commonly used image processing technique. Disadvantages and limitations of deep learning methods are specified in the paper [10].

Table 1: Conclusion Table

\begin{tabular}{|l|l|l|l|}
\hline Sr. No. & Published Year & Techniques/Algorithm used & Accuracy Percentage \\
\hline 1 & March 2011 & Otsu's Method \& Neural Network & $83-93 \%$ \\
\hline 2 & May 2014 & Generative Adversarial Networks & $91.42 \%$ \\
\hline 3 & Jan 2016 & Challenges in automatic plant diseases identification & - \\
\hline 4 & May 2016 & Caffe Framework \& CNN algorithm. & $96.3 \%$ \\
\hline 5 & Sept 2016 & Googlenet & $99.357 \%$ \\
\hline 6 & March 2017 & K-means clustering \& SVM classifier & $98.45 \%$ \\
\hline 7 & April 2017 & $\begin{array}{l}\text { Hotspot extraction(k means clustering) \& Navie } \\
\text { classifier }\end{array}$ & $869.67 \%$ \\
\hline 8 & Jan 2018 & Image Processing & $90-95 \%$ \\
\hline 10 & April 2018 & Deep Learning Techniques & $99.53 \%$ \\
\hline
\end{tabular}

\section{PROPOSED SYSTEM}




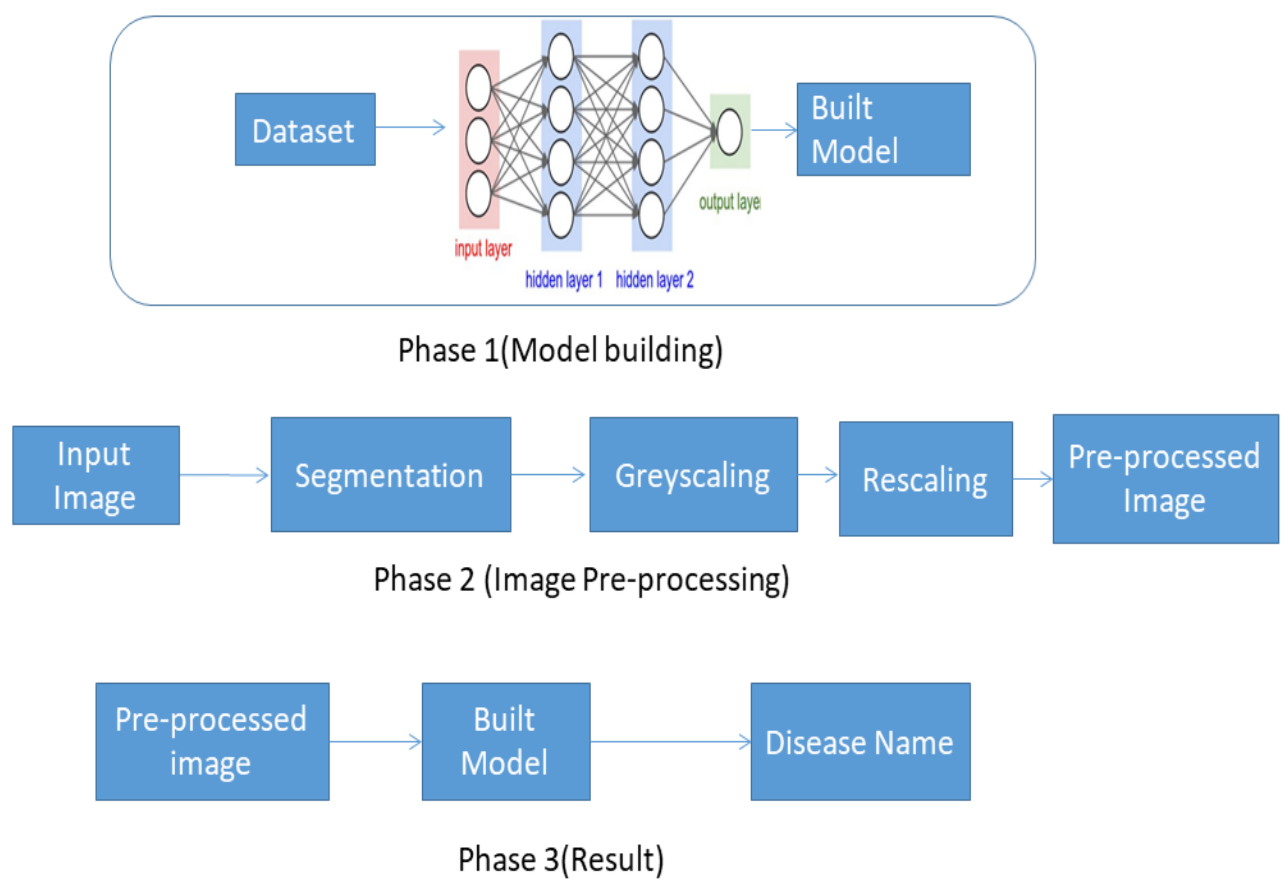

Steps in Proposed System:

Step 1: Importing dataset. Dataset is taken from Plantvillage Dataset.

Step 2: Training the dataset to build a model. Approximately $80 \%$ of dataset is provided for testing.

Step 3: Taking the input image from testing dataset which is approximately $20 \%$.

Step 4: Algorithms:

AlexNet, resNet

Image processing:

a. Segmentation

b. Greyscale

c. Rescale

Step 5: Pre-processed image.

Step 6: Feeding pre-processed image to "Built Model". Step 7: We will get results in terms of disease name.

\section{Proposed system in brief:}

First of all the most important thing was the data source, based on which we were going to implement the application. The dataset has been taken from an open source website called "PlantVillage.com", which provides different datasets of different crops. Among all other datasets, tomato crop image dataset has been selected as it is one of the common crops .

Secondly, conventional neural network (ConvNets or CNNs) was selected for image recognition and image classification. CNN is a class of deep neural network, which is most commonly applied for the usage of visual imagery. CNN uses different multilayer for feature selection. CNN takes input as an image, processes it and classify it according to the given class labels.

Inside $\mathrm{CNN}$, each input image of training and testing dataset will go through a series of convolution layer with filter, pooling, etc. We are using "Softmax" as an activation function in CNN. Inside the model different image pre-processing processes are happening which will minimize the further processing, will improve the image data and eliminate the unwanted noise and distortions. We are using Segmentation, grey-scaling and rescaling for the image pre-processing.

In segmentation, we partition the image into different regions, the main part of an image which is to be considered is taken into account, and the unwanted background is eliminated using techniques such as edge detection, region based detection. In grey-scaling, the 


\section{International Journal of Research in Advent Technology, Vol.7, No.2, February 2019 E-ISSN: 2321-9637 \\ Available online at www.ijrat.org}

segmented image is converted into grayscaled image, by converting into shades between white and black. Computers don't understand the image, but it understands in the terms of pixels. In rescaling, the greyscaled image is converted into arrays of pixels, and algorithms processes on such arrays of pixels. Image pre-processing is done in both training and testing phase.

After the model is built, we take the real-life image of a tomato crop and again on the leaf, several processes are done and we get output in the form of text format i.e. the disease name and the primitive steps to avoid/prevent the infected crop from disease.

\section{CONCLUSION}

Hence we concluded that among all techniques proposed by the authors in above papers, deep neural network gives very high accuracy. We will be implementing AlexNet and resNet algorithms which give more accurate results. On lab condition cultivated plant, but fail on real condition cultivated crop. So dataset should need more field images. For image preprocessing, we will be doing some basic steps like segmentation, greyscale and rescaling. In a result, we provide disease name and diagonsis of disease.

\section{REFERENCES}

[1] Heba AlHiary, Mohamad Hashem, "Fast and Accurate Detection and Classification of Plant Diseases", March 2011.

[2] Emanuel Cortes, "Plant Disease Classification using Convolutional Networks and Generative Adversarial Networks", 30 May 2014.

[3] Jayme Garcia, Arnal Barbedo, "A review on the main challenges in automatic plant disease identification based on visible range images", 29 Jan 2016.

[4] Srdjan Sladojevic, Marko Arsenovic, Andras Anderla, Dubravko Culibrk, Darko Stefanovic, "Deep Neural Networks based Recognition of Plant Diseases by Leaf Image Classification", 29 May 2016.

[5] Sharada P. Mohanty, David P. Hughes, Marcel Salathe, "Using Deep Learning for Image based Plant Disease Detection", 22 Sept 2016.

[6] Suja Radha, "Leaf Disease Detection Using Image Processing", March 2017.

[7] Alexander Johannes, Artzai Picon, Aitor AlvarezGilla, Jone Echazarra, Sergio RodriguezVaamonde, Ana Diez Navajas, "Automatic Plant Disease Diagnosis using Mobile Capture Devices Applied on a Wheat Use Case", 21 April 2017.
[8] Dixit Ekata Gajanan, Gavit Gayatri Shankar, Gode Vidya Keshav, "Android Based Plant Disease Identification System Using Feature Extraction Technique", Jan 2018.

[9] Konstantinos P. Ferentinos, "Deep Learning Medels for Plant Disease Detection and Diagnosis", 12 Feb 2018.

[10] Andeas Kamilaris,Francesc X. Prenafeta-Boldu, "Deep Learning in Agriculture: A Survey" 22 April 2018.

[11] H.M.Deshmukh, Jadhav Sanjivani, Lohar Utkarsha, Bhagat Madhuri, Salunke Shubhangi, "Plant Leaf Disease Identification System for Android", March 2016.

[12] M. Akila, P. Deepan, "Detection and Classification of Plant Leaf Diseases by using Deep Learning Algorithm", Jan 2018.

[13] Pramod S. Landge, Suhil A. Patil, Dhanashree S. Khot, Omkar D. Otari, Utkarsha G. Malavkar, "Automatic Detection and Classification of Plant Disease through Image Processing", July 2013.

[14] Jihen Amara, Bassem Bouaziz, Alsayed Algergawy, "A Deep Learning-based Approach for Banana Leaf Diseases Classification", March 2017.

[15] Kamlesh Golhani, Siva K. Balasundram, ganesan Vadamalai, Biswajeet Pradhan, "A review of neural networks in plant disease detection using hyperspectral data", April 2007.

[16] Savita N. Ghaiwat, Parul Arora, "Detection and Classification of Plant Leaf Diseases Using Image processing Techniques: A review", Feb 2014.

[17] Geng Ying, Li Miao, Yuan Yuan, Hu Zelin, "A Study on the Method of Image Pre-Processing for Recognition of Crop Diseases", March 2009.

[18] Jiazhi Pan, Min Huang, Yong He, "Crop and Weed Image Recognition by Morphological Operations and ANN Model", July 2007.

[19] G.Anthonys, N. Wickramarachchi, "An Image Recognition System for Crop Disease Identification of Paddy fields in Sri Lanka", Sept 2009.

[20] Jose Bermudez Castro, Raul Queiroz, Laura Cue La Rosa, Pedro Achanccarray Diaz, Ieda Sanches, "A Comparative Analysis of Deep Learning Techniques for Sub-tropical Crop Types Recognition from Multitemporal Optical/SAR Image Sequences", June 2017. 